\title{
Targeting resolvins in cholestatic liver injury
}

\author{
Luis A. Videla ${ }^{1}$, Rodrigo Valenzuela ${ }^{2}$ \\ ${ }^{1}$ Molecular and Clinical Pharmacology Program, Institute of Biomedical Science, Santiago, Chile; ${ }^{2}$ Department of Nutrition, Faculty of Medicine, \\ University of Chile, Santiago, Chile \\ Correspondence to: Rodrigo Valenzuela, PhD. Department of Nutrition, Faculty of Medicine, University of Chile, Santiago, Chile. \\ Email: rvalenzuelab@med.uchile.cl; rvalenzuelab@uchile.cl. \\ Comment on: Abshagen K, Hartmann A, Grüner L, et al. Limited potential of resolvin D1 in treatment of cholestatic liver fibrosis. Hepatobiliary Surg \\ Nutr 2020;9:587-96.
}

Submitted May 04, 2021. Accepted for publication May 15, 2021.

doi: 10.21037/hbsn-2021-15

View this article at: https://dx.doi.org/10.21037/hbsn-2021-15

In general terms, the acute inflammatory reaction in conditions of infection or tissue damage is a response of the immune system to cope with survival and return to homeostasis. Temporarily, the reaction is initiated by edema followed by the progressive accumulation of polymorphonuclear leukocytes (PMN) and monocytes that differentiate into macrophages, with production of proinflammatory mediators such as leukotrienes (e.g., LTB4) and prostaglandins (e.g., $\mathrm{PGE}_{2}, \mathrm{PGI}_{2}, \mathrm{PGF}_{2}$ ), which in excess may progress to chronic inflammation (1). Considering that the acute inflammatory response is a protective and selflimited phenomenon, its resolution is an active programmed reaction that is set in by specific pro-resolving mediators (SPMs), which are derived from n-3 polyunsaturated fatty acids (n-3 PUFAs). These include eicosapentaenoic acid (EPA) (C20:4n-3) and docosahexaenoic acid (DHA) $(\mathrm{C} 22: 6 \mathrm{n}-3)$ that are synthesized from the essential precursor $\alpha$-linolenic acid (ALA) (C18:3n-3), which in contact with resolving exudates generate specific types of SPMs, namely, resolvins, protectins and maresins (1). Besides n-3 PUFAs, the n-6 PUFA arachidonic acid (ARA) (C20:4n-6) also acts as a precursor for the synthesis of a specific type of SPMs the lipoxins, either by the action of 5-lipoxygenase (e.g., lipoxin $\mathrm{A}_{4}$ and $\mathrm{B}_{4}$ ) or triggered by aspirin-dependent cyclooxygenase-2 (COX-2) acetylation that forms an intermediary transformed by 5 -lipoxygenase into 15 -epilipoxin $\mathrm{A}_{4}(2)$. SPMs act in the $\mathrm{pM}$ to low $\mathrm{nM}$ range to interrupt PMN infiltration via signals that limit further PMN recruitment and tissue damage, with the concomitant enhancement of macrophage phagocytosis of apoptotic $\mathrm{PMN}$ s to attain resolution, the effective clearance of infecting microbes and tissue damage, with disappearance of the inflammatory exudate $(1,2)$.

Resolvin D1 (RvD1), the SPM studied by Abshagen et al. (3), is synthesized from DHA by the action of aspirinacetylated COX-2 and 5-lipoxygenase, although the process can proceed in the absence of aspirin, through the sequential catalysis of 15-lipoxygenase, 5-lipoxygenase and epoxide hydrolase (2). RvD1 exerts potent antiinflammatory (diminished PMN infiltration) and proresolving (enhanced macrophage phagocytosis) effects, the underlying protective mechanism of action being associated with the counteraction on oxidative stress-related tissue injury caused by PMN activation, which involves the production of reactive oxygen species (ROS) by NADPH oxidase and hypochlorite by myeloperoxidase, in addition to proteases (1). RvD1 signaling is mediated by lipoxin receptor ALX/FPR2 and G-protein coupled receptor 32 (GPR32), which downregulate the activation of nuclear factor- $\kappa \mathrm{B}(\mathrm{NF}-\kappa \mathrm{B})$, a redox-sensitive transcription factor that is central in inflammatory reactions (4).

Cholestatic liver diseases (CLDs) are related to bile transport obstruction conditions that slow biliary flow, which promote a cholestatic state with enhanced bile acid (BA) levels that induce BA cytotoxicity (5). The main cytotoxic mechanisms of BA include: (I) mitochondrial dysfunction associated with disruption of the mitochondrial membrane potential, enhancement in the production of ROS, with the consequent diminution in mitochondrial mass and DNA content altering respiratory functions; (II) ROS-dependent lipid peroxidation processes generating by-products that activate extracellular matrix cells and have direct 
fibrogenic effects on activated hepatic stellate cells (HSCs); and (III) activation of NF- $\mathrm{NB}$ inducing the expression of inflammatory cytokines (5). In this scenario, Abshagen et al. (3) studied the influence of $\mathrm{RvD} 1$ administration on cholestatic liver fibrosis in the model of bile duct ligation (BDL) in mice, by means of daily doses of $2 \mathrm{ng} \mathrm{RvD1/g}$ (total of 50-60 ng/mouse) for 2, 5 and 14 days. Data reported indicate the adequacy of BDL protocol used, that developed cholestatic liver injury, concomitantly with an inflammatory response, activation of HSCs and collagen expression, and a proliferative response of hepatocytes and non-parenchymal cells, with the consequent increase in liver weight/body weight ratio. However, RvD1 treatment failed to modify most of the studied parameters, except for a significant and early diminution in the activity of HSCs and in the deposition of extracellular matrix at 2 days after BDL (3). Some drawbacks are encountered in the commented article. First, the low effectiveness of the RvD1 treatment may be ascribed to insufficient levels attained in blood and liver, a parameter that was not determined by the authors, despite the availability of specific ELISA kits for RvD1 currently accessible $(5.0 \mathrm{pg} / \mathrm{mL}$ sensitivity; MyBioSource, California, USA; Thermo Fisher Scientific, Monza, Italy). Second, the lack of a previous dose-response study for RvD1 actions points to the possibility that doses higher than 50-60 ng/mouse (3) could have elicited significant results. In agreement with this prospect, (I) $100 \mathrm{ng} \mathrm{RvD} 1 /$ mouse three times per week via gavage from 5 to 21 days postinfection with $P$. aeruginosa, diminishes lung infection, inflammation and damage, promoting resolution in vivo in cystic fibrosis mice (6); (II) daily i.p. injections of 400-550 ng RvD1/rat for 21 days after a single dose of monocrotaline inducing right heart disease (RHD), prevents atrial fibrillation and suppresses inflammation and fibrotic/electrical remodelling by RHD (7); (III) low (100 ng/mouse) and high (300 ng/mouse) doses of RvD1 given in the last 2 weeks of a 4 weeks treatment with a methionine-choline deficient diet inducing non-alcoholic steatohepatitis (NASH), effectively improve liver oxidative stress, inflammation, steatosis and fibrosis, involving proinflammatory NF- $\mathrm{BB}$ downregulation and antioxidant Nrf2 activation (8); and (IV) using an ischemia/reperfusion injury protocol, the administration of $10 \mu \mathrm{g} \mathrm{RvD} 1 /$ mouse $10 \mathrm{~min}$ after reperfusion protects the kidney, as evidenced by the diminutions either in serum creatinine levels 24 and $48 \mathrm{~h}$ later, tissue fibrosis, leukocyte infiltration or macrophage activation (9). Interestingly, the administration of RvD1 alone in control experiments using 400-550 ng/rat (7) or $10 \mu \mathrm{g} /$ mouse (9) did not induce changes in any of the parameters measured. Consequently, in experimental settings evaluating SPM effects, assessment of pick time of effects and the levels of circulating and tissue RvD1 achieved are crucial for obtaining reliable results. This is especially important considering that RvD1 is effectively inactivated by an eicosanoid oxidoreductase (EOR), which elicits dehydrogenation at carbon-17 to produce an inactive 17-oxo-RvD1 metabolite (10).

At present time, the study of the co-supplementation with different SPMs on the outcome of CLDs or other pathologies has not been implemented in preclinical or clinical investigations. Compared to monotherapies, combined protocols represent relevant strategies for disease preclusion or resolution, as they might use lower doses of the agents and shorter supplementation periods than the monotherapies, and the similar or different underlying mechanisms of action might lead to synergistic or additive effects, thus minimizing side effects (11). This proposal is supported by recent studies in the high-fat diet model of obesity in mice, in which the co-administration of hydroxytyrosol, the potent antioxidant component of extra virgin olive oil, and the metabolic regulator DHA that increases the hepatic RvD1 and RvD2 levels in control and high fat diet (HFD)-fed mice, fully prevents liver steatosis (12) and the associated mitochondrial dysfunction (13), eluding disease progression into more irreversible stages lacking effective therapies at present time.

\section{Acknowledgments}

Funding: None.

\section{Footnote}

Provenance and Peer Review: This article was commissioned by the editorial office of Hepatobiliary Surgery and Nutrition. The article did not undergo external peer review.

Conflicts of Interest: Both authors have completed the ICMJE uniform disclosure form (available at https://hbsn. amegroups.com/article/view/10.21037/hbsn-2021-15/coif). The authors have no conflicts of interest to declare.

Ethical Statement: The authors are accountable for all aspects of the work in ensuring that questions related to the accuracy or integrity of any part of the work are appropriately investigated and resolved. 
Open Access Statement: This is an Open Access article distributed in accordance with the Creative Commons Attribution-NonCommercial-NoDerivs 4.0 International License (CC BY-NC-ND 4.0), which permits the noncommercial replication and distribution of the article with the strict proviso that no changes or edits are made and the original work is properly cited (including links to both the formal publication through the relevant DOI and the license). See: https://creativecommons.org/licenses/by-nc-nd/4.0/.

\section{References}

1. Serhan CN, Chiang N, Dalli J. The resolution code of acute inflammation: Novel pro-resolving lipid mediators in resolution. Semin Immunol 2015;27:200-15.

2. Bannenberg G, Serhan CN. Specialized pro-resolving lipid mediators in the inflammatory response: An update. Biochim Biophys Acta 2010;1801:1260-73.

3. Abshagen K, Hartmann A, Grüner L, et al. Limited potential of resolvin D1 in treatment of cholestatic liver fibrosis. Hepatobiliary Surg Nutr 2020;9:587-96.

4. Recchiuti A. Resolvin D1 and its GPCRs in resolution circuits of inflammation. Prostaglandins Other Lipid Mediat 2013;107:64-76.

5. Yokoda RT, Rodriguez EA. Review: Pathogenesis of cholestatic liver diseases. World J Hepatol 2020;12:423-35.

6. Isopi E, Mattoscio D, Codagnone M, et al. Resolvin D1 Reduces Lung Infection and Inflammation Activating Resolution in Cystic Fibrosis. Front Immunol 2020;11:581.

7. Hiram R, Xiong F, Naud P, et al. The inflammation-

Cite this article as: Videla LA, Valenzuela R. Targeting resolvins in cholestatic liver injury. HepatoBiliary Surg Nutr 2021;10(5):689-691. doi: 10.21037/hbsn-2021-15 resolution promoting molecule resolvin-D1 prevents atrial proarrhythmic remodelling in experimental right heart disease. Cardiovasc Res 2021;117:1776-89.

8. Li J, Deng X, Bai T, et al. Resolvin D1 mitigates nonalcoholic steatohepatitis by suppressing the TLR4MyD88-mediated NF- $\mathrm{kB}$ and MAPK pathways and activating the Nrf2 pathway in mice. Int Immunopharmacol 2020;88:106961.

9. Duffield JS, Hong S, Vaidya VS, et al. Resolvin D series and protectin D1 mitigate acute kidney injury. J Immunol 2006; 177:5902-11.

10. Sun YP, Oh SF, Uddin J, et al. Resolvin D1 and its aspirintriggered $17 \mathrm{R}$ epimer. Stereochemical assignments, antiinflammatory properties, and enzymatic inactivation. J Biol Chem 2007;282:9323-34.

11. Videla LA. Combined docosahexaenoic acid and thyroid hormone supplementation as a protocol supporting energy supply to precondition and afford protection against metabolic stress situations. IUBMB Life 2019;71:1211-20.

12. Soto-Alarcón SA, Ortiz M, Orellana P, et al. Docosahexaenoic acid and hydroxytyrosol coadministration fully prevents liver steatosis and related parameters in mice subjected to high-fat diet: A molecular approach. Biofactors 2019;45:930-43.

13. Ortiz M, Soto-Alarcón SA, Orellana P, et al. Suppression of high-fat diet-induced obesity-associated liver mitochondrial dysfunction by docosahexaenoic acid and hydroxytyrosol co-administration. Dig Liver Dis 2020;52:895-904. 